\title{
上海市居民居住迁移的区位选择 及其影响因素研究
}

\author{
崔 璨 $1,2,3$, 穆学英 ${ }^{2,3}$, 常鹤影 ${ }^{3}$, 李佳怡 ${ }^{3}$, 王丰龙 $1,2,4^{*}$ \\ (1. 华东师范大学中国行政区划研究中心, 上海 200241；2. 华东师范大学中国现代城市研究中心, 上海 200062; \\ 3. 华东师范大学城市与区域科学学院, 上海 $200241 ; 4$. 华南师范大学地理科学学院,广州 510631)
}

\begin{abstract}
摘 要: 随着中国住房改革的推进,城市居民通过居住迁移进行住房调整的行为日趋频繁。居住迁移作为城市空间 分异和重构的微观机制在城市地理学、住房研究中得到了广泛关注, 然而现有研究主要考察居住迁移的发生概率 和驱动因素, 较少论及居住迁移中的区位选择。论文基于 2018年“上海市居民住房和生活空间调查”问卷, 采用生 命历程和时间地理学视角, 刻画居民居住迁移的时空轨迹, 并使用多项逻辑斯蒂回归模型探究居住迁移前后居住 区位变化的影响因素。研究发现, 上海市居民居住迁移的主要区位选择为跨环线向外迁移,但不同世代、不同户 口、不同住房产权的居民的居住区位选择有较大差异。生命历程中的年龄、可达性中的工作单位区位以及住房属 性中的产权性质都是影响居住迁移区位选择的重要因素。
\end{abstract}

关键 词: 区位选择; 居住迁移; 住房产权; 时间地理学; 上海

居住迁移是指个人或家庭在一定空间范围内 (通常为同一城市内) 居住地点的变换,也是住房的 再选择过程。目前, 居住迁移在中国城市中已较为 频繁。根据 Li 等"对广州的调查研究, 2008-2012 年间, 31.2\%的广州市居民至少经历过 1 次居住迁 移。居住迁移伴随着一系列住房特征的变化。例 如, 居住迁移往往伴随居民住房所有权的获得。在 过去 $30 \mathrm{a}$ 的住房市场化改革进程中,中国大量城市 居民获得了自有住房一 2017 年中国家庭金融调 查(China Household Finance Survey, CHFS) 数据显 示中国城镇家庭住房自有率已经高达 $80.8 \%$ 。同 时,居民也在根据自身需求的变化变更居住区位。 如在郊区化的背景下,很多居民从城市中心搬到郊
区; 与此同时,也有很多住在郊区的家庭在子女接 近人学年龄时搬迁至教育资源相对集中的城市中 心。此外,居住迁移往往伴随着迁移者居住条件 ${ }^{[2]}$ 、 幸福感 ${ }^{[3-4]}$ 和健康 ${ }^{[5]}$ 的变化, 也是城市空间分异和重 构的微观机制 $[-7]$ 。

居住迁移是地理学重要的研究议题。自 Rossi 的经典著作 Why families move: $A$ study in the social psychology of urban residential mobility ${ }^{[8]}$ 出版以来, 西方地理学和住房研究领域的学者围绕居住迁移 的影响因素、迁移动机和迁移决策过程开展了大量 实证研究 ${ }^{[9-12]}$ 。国内地理学界也开展了一系列城市 内部居住迁移的研究 ${ }^{[13-17]}$ 。其中, 周春山 ${ }^{[18]}$ 是国内 最早开展城市内部居住迁移研究的地理学者, 他重

收稿日期: 2020-04-22; 修订日期:2020-07-22。

基金项目: 国家自然科学基金与荷兰研究理事会合作研究项目(72061137072); 国家自然科学基金项目(41701176); 上海市教 育发展基金会和上海市教育委员会“晨光计划”项目(18CG28)。[Foundation: Joint Fund from the National Natural Science Foundation of China and the Dutch Research Council, No. 72061137072; National Natural Science Foundation of China, No. 41701176; "Chenguang Program" Supported by the Shanghai Education Development Foundation and Shanghai Municipal Education Commission, No. 18CG28. ]

第一作者简介: 崔璨(1987-), 女, 安徽合肥人, 研究员, 主要研究方向为城市地理、社会地理。E-mail: ccui@geo.ecnu.edu.cn *通信作者简介: 王丰龙(1988-), 男, 研究员, 主要研究方向为政治地理学、幸福地理学和地理学思想。

E-mail: flwang@iud.ecnu.edu.cn

引用格式: 崔骤, 穆学英, 常鹤影, 等. 上海市居民居住迁移的区位选择及其影响因素研究 [J]. 地理科学进展, 2021, 40(3): 422-432. [Cui Can, $\mathrm{Mu}$ Xueying, Chang Heying, et al. Patterns and determinants of location choice in residential mobility: A case study of Shanghai. Progress in Geography, 2021, 40(3): 422-432. ] DOI: 10.18306/dlkxjz.2021.03.006 
点分析了居住迁移的影响因素以及居住迁移对城 市空间结构的影响。此后, 柴彦威、刘望保等学者 基于时间地理学和生命历程等视角, 研究了中国 城市居民居住迁移的空间特征、迁居原因和群体 分异 ${ }^{[19-23]}$ 。

居住迁移研究中一个重要议题即为居住区位 选择。国内外地理学界已经对此开展了系列研究, 着重分析了居民区位选择的影响因素 ${ }^{[24-27]}$ 。总体 上, 学者认为居住区位选择同时受城市层面和个人 层面因素的影响 ${ }^{[28]}$ 。相较于西方, 国内相关研究梳 理有限, 且已有的研究更偏重对城市层面因素的分 析, 从群体角度理解居住区位选择和居住空间分异 过程。如杜德斌等 ${ }^{[29]}$ 从不同收人、家庭结构、职业 和户口的居民的居住需求和住宅消费观念等角度 解释了深圳蛇口工业区的居住空间分异; 张文忠 等 ${ }^{[30]}$ 用居住选址空间偏好的差异解释了北京市的 居住空间分布格局; 周素红等 ${ }^{\left[{ }^{13}\right.}$ 则从居住一就业空 间匹配、城市空间拓展、阶层分化等角度剖析了广 州市居民居住区位选择的主要影响因素。而西方 研究更多从个体层面理解居住区位选择的动因, 并 重点考察个体生命历程、居住区位可达性以及住房 特征等因素的影响。(1)生命历程。不同年龄、代际 和生命历程阶段的居民住房偏好和区位选择存在 差异 ${ }^{[32]}$ 。如 Kim 等 ${ }^{[26]}$ 发现, 年龄以及是否需要照顾 子女会影响居民在居住迁移中对通勤距离的考 量。另外,有无子女(尤其是学龄子女)是影响居民 居住区位选择的重要因素 ${ }^{[3-35]}$ 。(2)可达性。众多研 究表明人们进行居住区位选择时会在工作地、公共 物品(如学校、治安、公共交通)、购物空间和休闲空 间的可达性之间做出取舍 ${ }^{[36-37]}$ 。其中, 工作地与居 住地之间的通勤时间是影响居住区位选择的重要 因素 ${ }^{[26,38-39]}$ 。由于有车居民和无车居民的出行能力 及其到达各类设施的难易程度存在巨大差异, 汽车 所有权和使用行为也是影响居住区位选择的重要因 素 ${ }^{[38,40-41]}$ 。(3) 住房特征。住房产权、社区类型、住房 品质及设施也是迁移决策中考虑的重要方面 ${ }^{[2]}$ 。如 很多家庭在选择居住区位时会同时考虑住房产权的 获得 ${ }^{[3-44]}$, 而拥有不同住房产权的居民在进行区位 选择时考量的因素也不尽相同 ${ }^{[4]}$ 。研究发现, 房价 和租金对居住区位选择有显著影响 ${ }^{[26,46-47]}$; 居住区 周边的教育资源及噪音水平等也是影响居住区位 选择的重要因素 ${ }^{[2,48]}$ 。

总的来说, 目前关于城市内部居住迁移和居住 区位选择的研究都已较多, 但是仍存在以下 3 个方
面局限。第一, 目前国内对居住迁移的研究更多关 注迁移行为或意愿的影响因素, 对居住迁移中区位 选择的研究偏少。虽然国外对于居住迁移的区位 选择研究相对比较成熟,但由于中国的政治经济环 境、城市发展阶段、住房供给体制、社会文化背景等 与西方存在较大差异, 国外的相关理论和实证结论 不一定适用于中国情境, 因此有必要对中国居民居 住迁移中的区位选择开展更多的实证研究。第二, 目前国内对居住区位选择影响机制的探究主要从 社会群体或城市空间结构分异的角度展开,较少从 个人尺度上进行分析。然而, 由于目前人们的住房 选择日益多元化, 群体的居住偏好和城市的住房供 给日益异质化,应该更多从个体尺度展开分析。第 三,目前国内对居住迁移中区位选择的调查数据和 分析方法尚不够多元。现有对居住区位选择的研 究大多基于截面数据(即仅对当前居所的区位进行 分析), 较少利用回溯性数据考察居民居住迁移的 区位选择。此外,尽管目前居住迁移的研究中已经 采用了生命周期或生命历程视角 ${ }^{[49-51]}$ 和时间地理学 方法 ${ }^{[20,52]}$, 但是这些研究更多在解释居住迁移行为 发生的概率或居住偏好, 鲜有研究将其用于分析居 住迁移前后的区位变化。为此,本文基于 2018 年上 海市居民住房与生活空间调查问卷, 采用生命历程 和时间地理学视角, 刻画居民居住迁移的时空轨 迹, 并结合西方相关研究的理论框架和中国国情, 在个人层面深人剖析居住迁移中居住区位选择的 影响因素。

\section{1 数据来源与研究方法}

\section{1 研究区域与数据来源}

本文采用的数据来源于研究团队 2018 年开展 的“上海市居民住房和生活空间调查”,该调查采取 多阶段的概率比例抽样法。首先, 在上海市建成区 范围内的 13 个区 $($ 不含远郊的金山区、奉贤区、崇明 区)进行分层抽样, 根据各街道/乡镇常住人口规模 随机等距抽取 32 个街道/乡镇作为初级抽样单元; 在抽中的街道/乡镇内, 将所辖居委会按常住人口规 模排序后随机抽选 1 个居委会; 再在每个抽中的居 委会内,按住宅小区名称排序, 随机抽取 1 2个住宅 小区; 最后, 在小区内, 依据楼栋和门牌号排序随机 等距抽选 35 户家庭(需在该小区居住半年以上), 对 年龄在 18 60 岁之间的户主或户主配偶进行访谈调 查。本次调查回收问卷 1127 份, 其中有效问卷为 
1052 份。该调查为回溯性调查, 采集内容不仅包括 被访者的户籍、年龄、性别、受教育程度、婚姻状况 等基本社会经济属性,还追溯收集了被访者的职业 生涯和住房生涯信息, 即被访者 18 岁后半年以上的 工作经历和居住经历的相关信息(经历起始年份、 区位, 以及工作/住房属性等)。笔者利用 ArcGIS、 百度地图 API等工具平台对被访者不同时期的居 住地址进行地理编码, 建立了具有时间属性的居住 空间数据集, 用于分析居住迁移的时空路径和区位 变化。图 1 展示了调研范围和调研小区的分布。

表 1 为调查样本的基本情况。可以发现, 受访 者性别较为均衡。根据出生年份可以将样本划分 为 4 个世代, 不同世代样本分布较为平均,但 80 后 占比稍多, 90 后因为年龄较小作为户主或户主配偶 的概率较低, 故在总样本中的占比较低。大部分受 访者都是已婚状态。问卷询问了受访者出生时和 调查时的户口状况，以此甄别上海本地人、新上海 人、外来人口 3 类人群。其中上海本地人占比最高, 为 $71.48 \%$; 新上海人较少, 只有 $5.42 \%$; 外来人口占 $23.10 \%$ 。从受教育程度来看, 本科及以上学历的受 访者占 $37.74 \%$ 。拥有中国共产党党员身份的受访 者占比 $12.17 \%$ 。从工作性质来看,党政机关、事业 单位等体制内工作者占 $36.03 \%$ 。超过一半的样本 家庭年收人在 10 万 30 万元之间。 $78.90 \%$ 的受访 者拥有自有产权住房。从居住区位分布看, $22.34 \%$

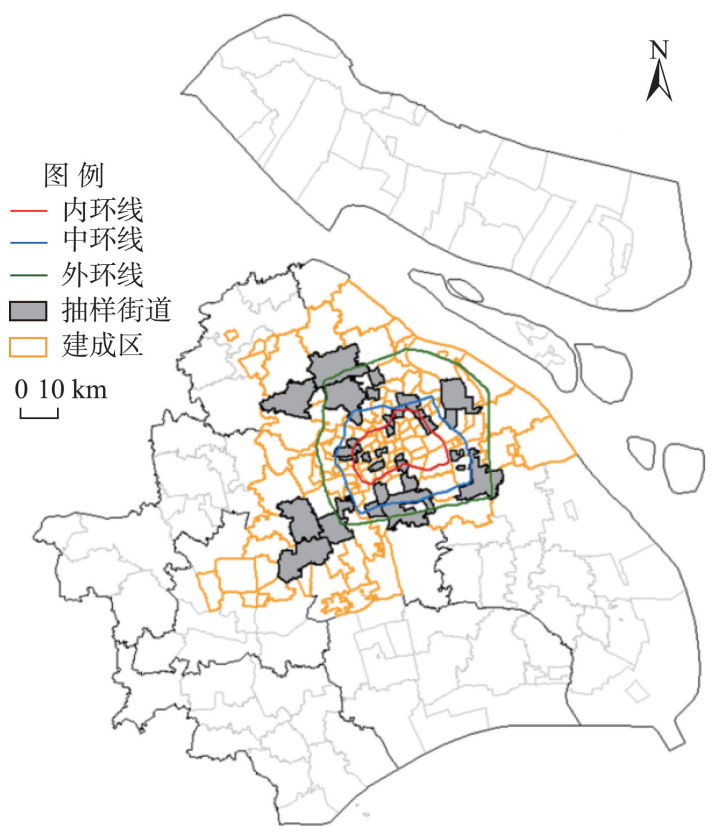

图 1 调研小区分布

Fig.1 Distribution of the sampled neighborhoods
的样本居住在内环以内, $34.03 \%$ 位于内环与中环 间, 28.04\%住在中环与外环间,居住在外环以外的 受访者占 $15.59 \%$ 。样本中具有迁居经历的样本为 457 个, 以迁居 1 次为主, 共计发生迁居事件 560 次。

\section{2 研究方法}

\subsection{1 时空路径可视化}

时空路径是时间地理学的基本概念和工具, 是 分析个体活动参与和活动机会限制的有效框架, 经 常被用来记录个体日常活动或居住迁移在时空中 的轨迹 ${ }^{[53]}$ 。时空路径是由二维地理平面中的一个 空间坐标点和一个时间轴上的点共同构成的三维 空间中的轨迹。本文采用 ArcGIS 软件和 Space-

表 1 调查样本基本情况

Tab.1 Sample profile

\begin{tabular}{|c|c|c|c|}
\hline 属性 & 类别 & 频数 & 占比/\% \\
\hline \multirow[t]{2}{*}{ 性别 } & 男 & 481 & 45.72 \\
\hline & 女 & 571 & 54.28 \\
\hline \multirow[t]{4}{*}{ 世代 } & 60 后 & 253 & 24.05 \\
\hline & 70 后 & 314 & 29.85 \\
\hline & 80 后 & 361 & 34.31 \\
\hline & 90 后 & 124 & 9.79 \\
\hline \multirow[t]{2}{*}{ 婚姻状况 } & 已婚 & 832 & 79.09 \\
\hline & 未婚、离异或丧偶 & 220 & 20.91 \\
\hline \multirow[t]{3}{*}{ 户口 } & 上海本地人 & 752 & 71.48 \\
\hline & 新上海人 & 57 & 5.42 \\
\hline & 外来人口 & 243 & 23.10 \\
\hline \multirow[t]{2}{*}{ 户口性质 } & 城镇 & 909 & 86.41 \\
\hline & 农村 & 143 & 13.59 \\
\hline \multirow[t]{2}{*}{ 学历 } & 本科以下 & 655 & 62.26 \\
\hline & 本科及以上 & 397 & 37.74 \\
\hline \multirow[t]{2}{*}{ 是否中共党员 } & 是 & 128 & 12.17 \\
\hline & 否 & 924 & 87.83 \\
\hline \multirow[t]{2}{*}{ 工作性质 } & 体制内 & 379 & 36.03 \\
\hline & 其他 & 673 & 63.97 \\
\hline \multirow[t]{3}{*}{ 家庭年收人 } & 10 万元以下 & 333 & 31.65 \\
\hline & 10 万 30 万元 & 575 & 54.66 \\
\hline & 30 万元以上 & 144 & 13.69 \\
\hline \multirow[t]{2}{*}{ 住房产权 } & 自有产权 & 830 & 78.90 \\
\hline & 其他 & 222 & 21.10 \\
\hline \multirow[t]{4}{*}{ 居住区位 } & 内环以内 & 235 & 22.34 \\
\hline & 内环与中环间 & 358 & 34.03 \\
\hline & 中环与外环间 & 295 & 28.04 \\
\hline & 外环以外 & 164 & 15.59 \\
\hline \multirow[t]{4}{*}{ 居住迁移次数 } & 0 次 & 595 & 56.56 \\
\hline & 1 次 & 372 & 35.36 \\
\hline & 2 次 & 67 & 6.37 \\
\hline & 3 次及以上 & 18 & 1.71 \\
\hline
\end{tabular}


Time Path 插件对个体居住迁移的时空路径进行可 视化。图 2 是一个居住迁移时空路径的示例, 如果 个体的居住地点不发生变动, 其时空路径为一条垂 直于地图的线段; 如果发生居住迁移时, 其时空路 径表现为一条平行于地图的水平线。图中该个体 在第一个居住地的居住经历从其出生开始, 先后迁 居2次。

\subsection{2 多项逻辑斯蒂回归模型}

本文运用类别变量描述居住迁移的区位变 化。具体而言, 本文将区位变化分为 5 种类型 : (1) 搬迁前、后都居住在内环以内, (2) 搬迁前、后都居住 在内环与中环之间, (3) 搬迁前、后都居住在中环之 外(中环与外环之间以及外环以外), (4) 跨环线向内 搬迁,(5) 跨环线向外搬迁。鉴于区位变化是多分类 变量, 本文采用已有研究 ${ }^{[1,34,54-56]}$ 普遍使用的多项逻 辑斯蒂回归模型分析上海市居民居住迁移区位变 化的影响因素。其中, 因变量为 5 种居住迁移区位 变化类型, 搬迁前、后都居住在中环之外(中环与外

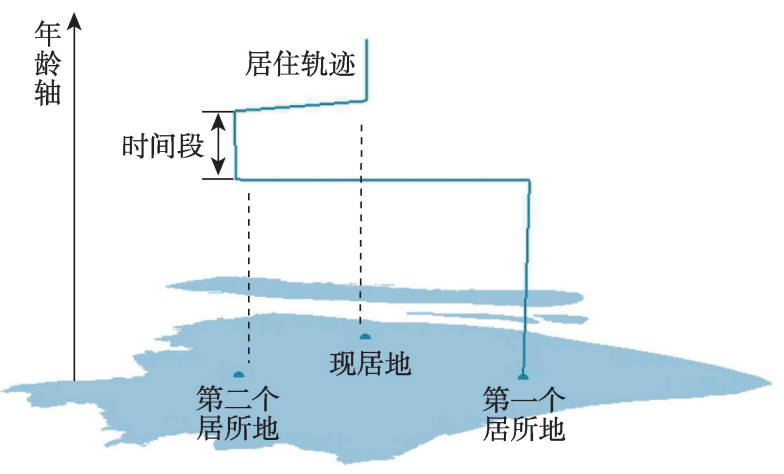

图 2 居住轨迹时空路径三维可视化示例

Fig.2 An illustration of the space-time path of residential trajectory

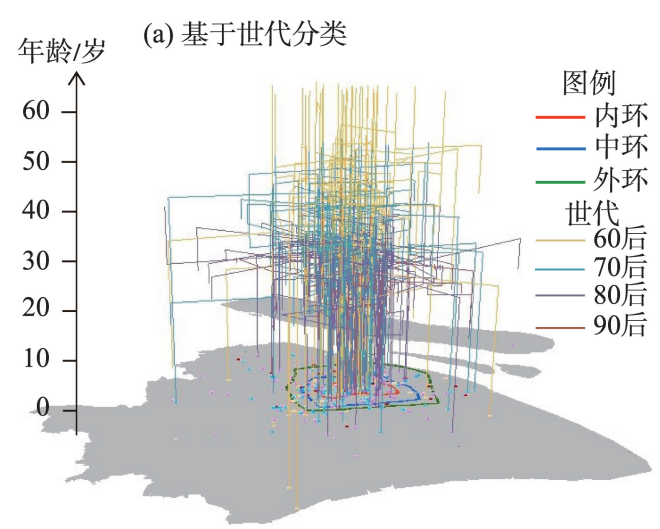

环之间以及外环以外)这一类型为参照组 $(J)$, 第 $j$ 类 区位变化与参照组区位变化的似然比符合如下对 数线性方程:

$$
\ln \left(\frac{P(y=j \mid X)}{P(y=J \mid X)}\right)=\alpha_{j}+\sum_{i=1}^{n} \beta_{j i} x_{i}
$$

发生第 $j$ 类区位变化类型的概率为:

$$
P(y=j \mid X)=\frac{\exp \left(\alpha_{j}+\sum_{i=1}^{n} \beta_{j i} x_{i}\right)}{1+\sum_{j=1}^{J-1} \exp \left(\alpha_{j}+\sum_{i=1}^{n} \beta_{j i} x_{i}\right)}
$$

式中: $\alpha_{j}$ 是常数项回归系数; $x_{i}$ 表示模型中引人的 $n$ 个自变量, 根据现有文献主要包括生命历程、可达 性、住房属性、个人社会经济属性等相关变量; $\beta_{j i}$ 是 自变量 $x_{i}$ 的系数。因为同一个体/家庭可能发生多 次迁居事件, 为此, 模型中使用聚类稳健标准差以 修正同一个体/家庭多次迁移的相关性 ${ }^{[7]}$ 。

\section{2 居住迁移的时空特征}

图 3 展示了基于世代和户口分类的上海市居民 居住迁移的时空轨迹。将上海市的地理空间压缩 成二维平面, $z$ 轴为迁居时的年龄, 构建了三维居住 轨迹图。整体来看, 居住迁移从 20 岁开始逐渐增 多, 在 30 岁左右达到顶峰, 随后呈下降趋势, 并在 50 岁左右保持在较低的水平。上海市居民的居住 迁移方向以自市中心向四周扩散为主, 基本反映了 20 世纪 90 年代以来上海市居住空间的郊区化趋 势,也与已有关于上海市居住和就业郊区化的研究 发现基本一致 ${ }^{[58-59]}$ 。

不同世代居民的迁移时空轨迹(包括迁居起始 年龄和迁居方向)存在明显差异,这与不同世代所

(b) 基于户口分类

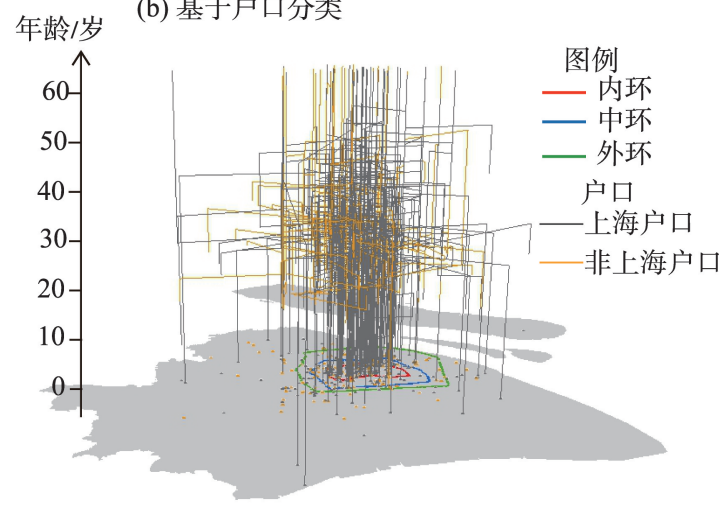

图 3 上海市居民居住迁移时空轨迹

Fig.3 Spatiotemporal trajectory of residential mobility in Shanghai by generation and population registration types 
处的住房市场制度环境和城市空间结构紧密相 关。对于 60 后而言, 受福利住房分配制度影响, 他 们年轻时发生居住迁移的频率极低, 直到 40 岁以 后, 随着住房市场化改革, 其迁居行为才渐渐增多, 且多为从远郊区向市中心的长距离迁移, 反映了城 镇化早期人口向建成区聚集的现象。对于 70 后而 言, 迁移发生的年龄有所提前, 主要集中在 30 岁左 右, 迁移方向多为从郊区向市中心。而 80 后群体居 住迁移更加年轻化, 在 25 岁左右即已出现频繁的居 住迁移, 迁居方向在城市郊区化的大背景下也更多 表现为从市中心向郊区迁移。90后群体由于观测 时间较短, 大部分样本只存在一次居住迁移经历, 且迁移的起止点主要在城市中心。

上海市本地人口与外来人口居住迁移轨迹的 差异也较为显著。如图 $3 \mathrm{~b}$ 所示, 相较于上海户口居 民, 外来人口居住迁移的起始年龄和迁移方向都更 为集中, 这往往与他们在特定年龄阶段居住于上海 的情况(一般成年后来到上海, 中老年后离开上海) 有关。上海本地人口的迁居大多始于 18 岁, 在 30 岁左右迁移数量达到高峰, 且随着年龄的增加迁移 率仍然很高, 40 岁之后仍有相当一部分的本地人口 进行居住迁移, 可以理解为本地人口持续通过居住 迁移在改善住房条件。而外来人口在上海的住房 生涯多始于 20 岁之后, 在 25 岁左右迁移次数明显 增加, 且在 40 岁之后逐渐减少。从迁移方向看, 本 地人口在迁移中更多占据优越的居住区位, 在内环 内迁移或跨环线向内迁移的比例较高; 而外来人口 受限于房价和迁移成本, 呈现出中环以外就近迁移 和跨环线向外迁移并存的特征。

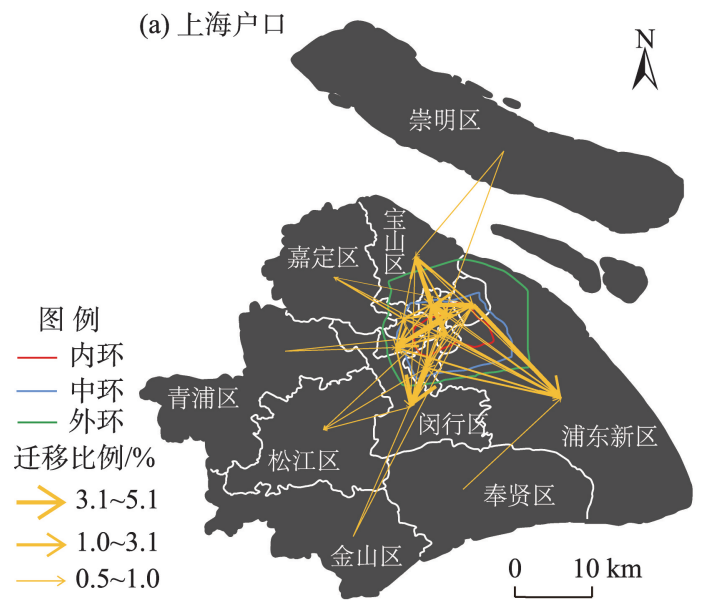

图 4更明确地呈现了上海户口和非上海户口居 民的跨区迁移路径。可以发现, 本地人口迁移前后 的区位多集中于中心城区,但向城市郊区迁移尤其 是向浦东、闵行和宝山迁移的数量也较多。数据显 示本地人口迁出地排名前三的为杨浦区、浦东新区 和徐汇区, 主要迁人地为浦东新区、静安区和杨浦 区。相较而言, 外来人口的区位分布较为分散,向 郊区曼延明显。浦东新区和闵行区为其主要的迁 出地, 而浦东新区、杨浦区和远郊的松江区为其主 要迁人地。

进一步对居住迁移前后的区位变化与产权变 化进行交叉分析,并比较本地人口和外来人口的差 异(图 5)。从区位变化看,上海本地人口与外来人口 的居住区位均呈现出多样化的发展态势, 同环线内

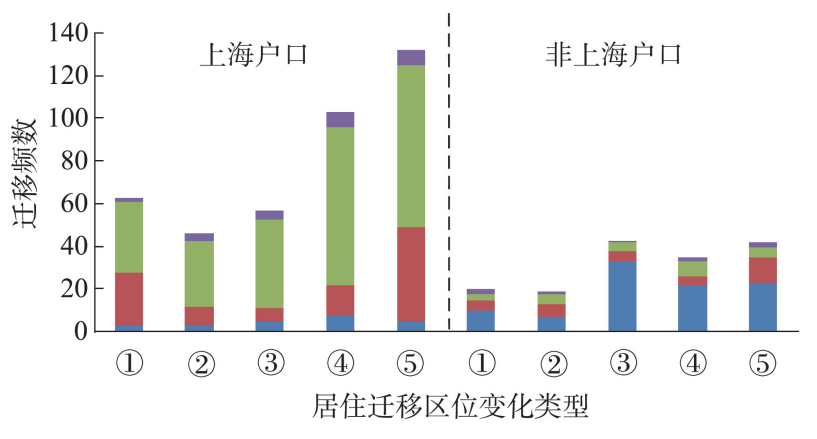

- 自有转租赁 - 持续自有型 $\square$ 租赁转自有 - 持续租赁型

注: (1) 搬迁前、后都居住在内环以内 ; (2) 搬迁前、后都居住在 内环与中环之间; (3) 搬迁前、后都居住在中环之外; (4) 跨环线向内 搬迁;(5) 跨环线向外搬迁。

图 5 居住区位与产权变化

Fig.5 Frequency of residential location changes across the ring roads

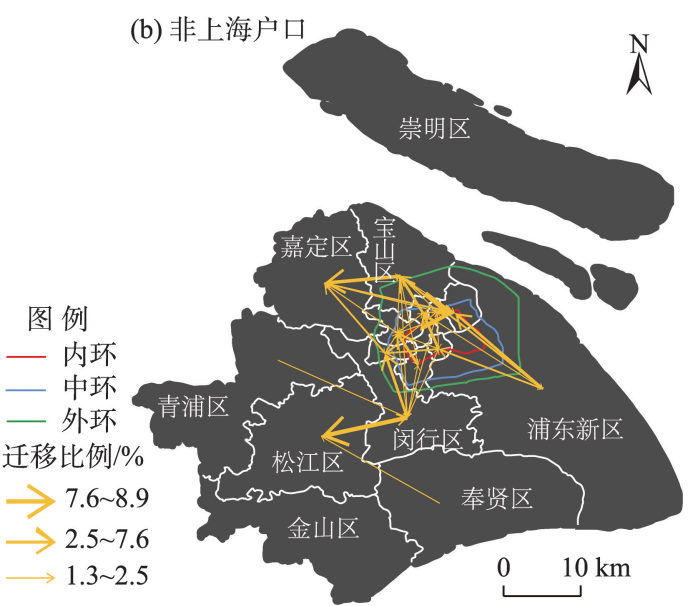

图 4 上海市居民跨区迁移流向

Fig.4 Flows of inter-district residential moves in Shanghai by population registration types 
部迁移与跨环线迁移并存, 跨环线向内与向外迁移 2 个方向共存。同时, 2 个群体在居住区位选择上也 存在明显的差别。上海本地人口以跨环线迁移为 主, 其中跨环线向外迁移稍多于跨环线向内迁移, 在同一环线区域内部迁移的样本分布较为均匀。 外来人口的迁移则以在中环以外、跨环线向外迁移 为主, 跨环线向内迁移也占一定比例, 但在内环以 内、中环以内迁移的较少。基于迁移前后的产权性 质, 可将产权变化分为以下 4 种情况: 持续租赁型、 持续自有型、租赁转自有、自有转租赁。上海户口 和非上海户口居民在产权变化上的差异更加明显, 上海本地居民以持续自有型即自有产权住房改善 型迁移为主,而外来人口主要以持续租赁型为主。 结合居住迁移的区位变化和产权变化, 可以发现, 相较于外来人口而言, 本地人口住房权属由租赁向 自有转变更容易发生在内环以内。另外, 可能由于 持续租赁型的外来人口对住房成本较为敏感, 较难 负担中环以内的房租, 故多集中在中环以外。

\section{3 居住迁移区位选择的影响因素}

本文使用多项逻辑斯蒂回归模型探究居住迁 移区位选择的影响因素。模型结果显示, 生命历 程变量中, 年龄(这里主要采用出生世代衡量)与居 住迁移的区位选择密切相关(表 2)。60后一直在内 环居住的概率较高, 而 80 后和 90 后更可能居住在 城市中环以外的地区。住房市场化改革以及城市 空间重构对于不同世代的居住空间选择有重要影 响。中老年世代(70 后及以前人群)在城市发展早 期得以通过单位分房聚居在城市中心; 伴随着城 市的郊区化以及住房价格的快速上涨, 年轻世代 (80 后及以后人群)更多主动或被动选择在中环以 外居住。然而, 结婚、生子等生命历程事件对迁居 的区位选择并无显著影响, 这可能是因为这些生 命历程事件的影响已经体现在世代变量中,也可 能是由于上海市不同居住区位无法完全满足伴随 不同生命历程事件的需求变化(如虽然人们在结婚 和子女出生后更倾向于居住在有较多绿地和较少 机动车的郊区, 但是由于好的学区集中在中心城 区, 因此在结婚或养育小孩子时往往仍选择住在 中心城区)。

在可达性变量中, 工作单位所在地与居住迁移 的区位选择紧密相关。与居住区位的划分类似, 本
文将工作地按环线划分为 3 类: 内环以内、内环与中 环间、中环以外。从模型结果看,工作单位处于内 环至中环间的居民相较于在内环工作的居民更不 会居住在内环以内; 换言之, 在内环以内工作的居 民更有可能居住在内环。职住距离是影响居民居 住迁移区位选择的重要因素,通勤的便利使其愿意 承担住在内环较高的住房成本。工作地位于中环 以外的居民在迁居前后都更有可能住在中环以 外。与西方已有的研究结论不同 ${ }^{[40-41]}$, 是否拥有私 家车与上海市居民居住迁移的区位选择并无显著 关联, 这可能是由于上海市住房购置成本远高于汽 车购置成本, 因此是否有车对于能否搬往一些偏远 的居住区位影响不大, 而更可能是搬迁到郊区后才 会根据需求购车。另外,上海的私家车牌照拍卖制 度也可能使得是否拥有私家车对居住区位选择的 影响与西方国家不同。

在住房属性变量中,产权的变更与居住迁移的 区位变化显著相关。具体而言,那些在迁移过程中 实现住房权属由租赁向自有转变的群体,其居住区 位变化大多以一直在内环以内或跨环线向内迁移 为主。这一发现与已有研究发现有所不同。一般 认为, 人们在做出住房决策行为时会在产权与区位 之间进行权衡, 即可能为了获得住房产权而选择相 对偏远、房价较低的区位。如Fang 等 ${ }^{[49]}$ 发现北京市 购房的群体更多选择在近郊区而较少选择在中心 区的区位。然而,本文研究发现上海居民在居住迁 移过程中新获得住房产权的群体更多保留了在中 心区的优势区位或实现了向内迁移的区位提升。 这说明一部分上海居民能够同时实现住房产权与 住房区位在住房阶梯(housing ladder)中的上升, 这 对于理解伴随着居住迁移的住房分异具有重要意 义,值得未来的研究进一步关注。不过,社区类型 对居住迁移的区位选择没有显著影响,说明是否为 商品房小区并非人们住房区位选择的主要因素。

本文还进一步控制了个人的人口社会经济属 性的影响。与描述性分析一致, 不同的户口属性呈 现出了不同的居住区位变化。相较于在较早年代 就在城市中心拥有住房的本地人口, 新上海人在居 住迁移区位选择上的劣势凸显, 他们很少居住在内 环以内, 而更可能居住在中环以外。家庭年收人高 的群体在居住区位上优势凸显,家庭年收人 30 万元 以上的群体居住在内环以内的概率和跨环线向内 迁移的概率显著高于家庭年收人小于 10 万元的群 
表 2 居住迁移区位变化的多项逻辑斯蒂模型 (参考组: 中环以外 $\rightarrow$ 中环以外)

Tab.2 Results of multinomial logistic regression model on location choice in residential mobility

\begin{tabular}{|c|c|c|c|c|}
\hline 变量 & 内环以内 & 内环与中环间 & 跨环线向内 & 跨环线向外 \\
\hline \multicolumn{5}{|l|}{ 生命历程 } \\
\hline \multicolumn{5}{|l|}{ 世代(参照组: 60后) } \\
\hline 70 后 & -0.520 & -0.333 & -0.237 & 0.154 \\
\hline 80 后 & $-1.342^{* *}$ & -0.826 & $-0.951^{* *}$ & -0.484 \\
\hline 90 后 & $-1.508^{*}$ & -1.207 & $-1.501^{* *}$ & -0.698 \\
\hline 已婚 & -0.192 & 0.620 & -0.531 & -0.090 \\
\hline 有孩子 & -0.698 & -0.621 & -0.381 & -0.684 \\
\hline \multicolumn{5}{|l|}{ 可达性 } \\
\hline \multicolumn{5}{|l|}{ 工作区位(参照组: 内环以内) } \\
\hline 内环与中环间 & $-1.439^{* *}$ & -0.048 & -0.745 & -0.583 \\
\hline 中环以外 & $-3.265^{* * *}$ & $-1.935^{* * *}$ & $-2.060^{* * *}$ & $-1.821^{* * *}$ \\
\hline 汽车所有权(参照组: 无车) & -0.033 & -0.246 & 0.532 & 0.267 \\
\hline \multicolumn{5}{|l|}{ 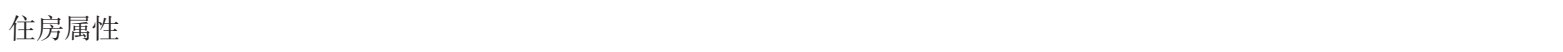 } \\
\hline \multicolumn{5}{|l|}{ 产权变化(参照组: 持续租赁型) } \\
\hline 自有转租赁 & 0.114 & 0.450 & 0.180 & 0.304 \\
\hline 租赁转自有 & $1.156^{*}$ & 1.010 & $1.057^{* *}$ & -0.077 \\
\hline 持续自有型 & -0.106 & 0.306 & -0.096 & -0.123 \\
\hline \multicolumn{5}{|l|}{ 小区类型(参照组:其他) } \\
\hline 商品房 & 0.108 & 0.405 & -0.044 & 0.343 \\
\hline \multicolumn{5}{|l|}{ 控制变量: 个人的人口社会经济属性 } \\
\hline 性别(参照组:男性) & 0.494 & 0.422 & 0.293 & 0.166 \\
\hline \multicolumn{5}{|l|}{ 户口(参照组: 上海本地人) } \\
\hline 新上海人 & $-11.688^{* * *}$ & -0.153 & -1.228 & -0.336 \\
\hline 外来城镇人口 & 0.292 & 0.148 & 0.221 & 0.019 \\
\hline 外来农村人口 & -0.140 & -0.373 & -0.257 & $-0.906^{*}$ \\
\hline \multicolumn{5}{|l|}{ 家庭年收人(参照组: 小于 10 万元) } \\
\hline 10 万 30 万元 & $1.114^{* *}$ & 0.138 & 0.461 & 0.469 \\
\hline 30 万元以上 & $2.461^{* * *}$ & 1.075 & $1.293^{* *}$ & $1.660^{* * * *}$ \\
\hline 职业(单位负责人及专业技术人员) & -0.807 & $0.881^{* *}$ & -0.258 & 0.104 \\
\hline 工作性质(体制内) & 0.670 & 0.493 & $0.666^{*}$ & 0.254 \\
\hline 教育水平(本科及以上) & -0.180 & 0.318 & 0.249 & -0.038 \\
\hline 中共党员(参照组: 非中共党员) & $-2.819^{* *}$ & -0.082 & -0.313 & -0.019 \\
\hline 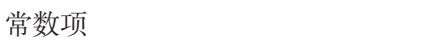 & 1.156 & -0.396 & $1.753^{* *}$ & 1.229 \\
\hline 有效样本数 & \multicolumn{4}{|c|}{560} \\
\hline Pseodu $R^{2}$ & \multicolumn{4}{|c|}{0.141} \\
\hline Loglikelihood & \multicolumn{4}{|c|}{-744.889} \\
\hline Wald 检验(chi2) & \multicolumn{4}{|c|}{1104.280} \\
\hline
\end{tabular}

注:******分别表示 $P<0.10 、 P<0.05 、 P<0.01$ 。

体。从职业类型看, 被认为具有较高社会地位的管 理者和专业技术人员比从事其他职业的人员更可 能居住在内环与中环之间。体制内工作者因体制 内优势(房改房、集资房、住房补贴和住房公积金等) 更有可能进行资本积累, 从而实现跨环线的向内迁 移。相较而言, 中共党员在居住迁移区位上的优势 并不凸显, 他们不太可能一直居住在内环以内。

\section{4 结论与讨论}

本文基于 2018 年开展的“上海市居民住房和生 活空间调查”,利用时间地理学的时空路径可视化 方法和多项逻辑斯蒂回归模型系统分析了上海市 居民居住迁移中的区位选择模式及其影响因素。 研究发现: 1) 上海市居民居住迁移以跨环线向外迁 
移为主,但不同世代、不同户口、不同住房产权的居 民在居住迁移中呈现出显著不同的时空特征。相 较于中老年世代而言, 年轻世代迁居起始年龄普遍 较小, 且呈现出由市中心向郊区迁移的趋势; 相较 于上海户口居民, 外来人口的迁移年龄和迁居方向 更为集中; 住房权属实现由租赁向自有转变的居 民, 其在居住区位上往往容易跨环线向外迁移。2 影响居住迁移区位选择的因素中, 个人的生命历 程、可达性和住房属性均发挥着重要作用。具体而 言: 中老年世代(70后及以前人群)的迁居主要集中 在城市中心地区, 而年轻世代( 80 后及以后人群)更 可能在城市中环以外进行居住迁移, 结婚、生子等 生命历程事件对区位选择无显著影响; 可达性因素 中,工作单位的区位与居住区位呈现较高一致性, 通勤仍是人们在进行居住区位选择的重要影响因 素, 而是否拥有私家车对居住迁移区位选择的影响 不大; 住房属性中的产权与居住迁移的区位变化显 著相关, 部分居民在居住迁移中实现了住房产权获 得和住房区位的同时改善。

本文的主要贡献体现在以下 3 个方面。首先, 尝试将居住迁移和居住区位选择的研究整合到一 起, 拓展了地理学对住房选择行为的研究。已有对 居住迁移的研究偏重从人口地理或城市地理的角 度分析人们居住迁移决策的影响因素及其对城市 空间结构的影响, 但是很少关注人们在居住迁移中 居住区位的变化特征及其影响因素。第二, 基于现 有文献构建了一个包含生命历程、可达性和住房特 征的系统分析居住迁移中居住区位选择影响因素 的分析框架。这一框架能够用于未来对居住区位 选择影响因素的进一步研究。最后, 基于实地调查 数据, 对上海市居住迁移的居住区位变化特征和影 响因素进行了初步分析, 发现了与以有研究不同的 结论, 如住房权属和住房区位的变化方向在住房阶 梯中具有一致性。

受数据限制, 本文还有以下 3 个方面值得未来 深人开展的研究方向。首先, 出于模型简洁和数据 可得性的考虑, 本文解释居住迁移中区位选择影响 因素主要基于多项逻辑斯蒂回归模型。不过, 考虑 到部分居民的居住迁移决策是在不同限制条件下 权衡形成的, 因此未来研究可以进一步使用嵌套逻 辑斯蒂回归、门槛模型等其他模型, 更精细地刻画 迁移中的区位选择机理。此外, 本文在提供了一个 相对完整的影响因素分析框架的同时, 由于同时涉
及多个指标而无法对个别因素的因果关系(如婚姻 或工作可达性等)进行更深人、稳健的分析,未来可 以基于倾向性评分匹配和准实验设计(如观察同等 条件下工作地可达性发生不同变化的 2 类群体之间 在居住迁移区位选择上的差异)对本文分析结论的 因果关系进行稳健性检验。第二,本文目前只是基 于城市环线对居住区位进行衡量和划分,未来研究 可以从小区类型、小区周边不同设施的可达性、迁 移距离等其他指标丰富和补充本文对居住区位的 刻画及其影响因素的分析。第三,本文仅对上海近 $20 \mathrm{a}$ 来居住迁移中区位选择进行了分析,未来研究 可以覆盖更长的时段和更多的城市, 从而检验本文 的结论,并比较不同历史阶段和地域居民居住迁移 中的居住区位变化及其影响因素的差异。

\section{参考文献(References)}

[1] Li S M, Mao S Q. The spatial pattern of residential mobility in Guangzhou, China [J]. International Journal of Urban and Regional Research, 2019, 43(5): 963-982.

[2] Wu F L. Residential relocation under market-oriented redevelopment: The process and outcomes in urban China $[\mathrm{J}]$. Geoforum, 2004, 35(4): 453-470.

[3] Wang F. Home relocation and changes in satisfaction with residence, travel and life: A study of Beijing [D]. Hongkong, China: Hong Kong Baptist University, 2015.

[4] Wang F L, Wang D G. Changes in residential satisfaction after home relocation: A longitudinal study in Beijing, China [J]. Urban Studies, 2020, 57(3): 583-601.

[5] 李春江, 马静, 柴彦威, 等. 居住区环境与噪音污染对居 民心理健康的影响: 以北京为例 [J]. 地理科学进展, 2019, 38(7): 1103-1110. [Li Chunjiang, Ma Jing, Chai Yanwei, et al. Influence of neighborhood environment and noise pollution on residents' mental health in Beijing. Progress in Geography, 2019, 38(7): 1103-1110. ]

[6] 刘小平, 黎夏, 陈逸敏, 等. 基于多智能体的居住区位空 间选择模型 [J]. 地理学报, 2010, 65(6): 695-707. [Liu Xiaoping, Li Xia, Chen Yimin, et al. Agent-based model of residential location. Acta Geographica Sinica, 2010, 65 (6): 695-707. ]

[7] 王铮, 翟石艳. 计算机实验人文地理学的理论构建及应 用: 以城市居住区位选择为例 [J]. 地理科学进展, 2013, 32(10): 1444-1453. [Wang Zheng, Zhai Shiyan. Theoretical framework and application of computer-aided experimental human geography: Case study of urban residential location choice. Progress in Geography, 2013, 32(10): 1444-1453. ] 
[8] Rossi P H. Why families move: A study in the social psychology of urban residential mobility [M]. Glencoe, USA: Free Press, 1955

[9] Clark W A V. Recent research on migration and mobility: A review and interpretation [J]. Progress in Planning, 1982, 18: 1-56.

[10] Coulter R, van Ham M, Findlay A M. Re-thinking residential mobility: Linking lives through time and space [J]. Progress in Human Geography, 2016, 40(3): 352-374.

[11] Morris T, Manley D, Sabel C E. Residential mobility: Towards progress in mobility health research [J]. Progress in Human Geography, 2018, 42(1): 112-133.

[12] Quigley J M, Weinberg D H. Intra-urban residential mobility: A review and synthesis [J]. International Regional Science Review, 1977, 2(1): 41-66.

[13] 柴彦威, 田原裕子, 李昌霞. 老年人居住迁移的地理学 研究进展 [J]. 地域研究与开发, 2006, 25(3): 109-115. [Chai Yanwei, Tahara Yuko, Li Changxia. A review of the geographical research on the elderly migration. Areal Research and Development, 2006, 25(3): 109-115. ]

[14] 秦萧, 甄峰. 信息渠道对城市居民迁居空间的影响: 以 南京为例 [J]. 地理研究, 2016, 35(10): 1846-1856. [Qin Xiao, Zhen Feng. The impacts of information channels on moving space: A case study on Nanjing. Geographical Research, 2016, 35(10): 1846-1856. ]

[15] 周春山, 许学强. 西方国家城市人口迁居研究进展综述 [J]. 人文地理, 1996, 11(4): 23-27. [Zhou Chunshan, Xu Xueqiang. A summary of research progress on residential mobility in western cities. Human Geography, 1996, 11 (4): 23-27. ]

[16] 杨振山, 吴笛, 杨定. 迁居意愿、地方依赖和社区认同: 北京中关村地区居住选择调查分析 [J]. 地理科学进 展, 2019, 38(3): 417-427. [Yang Zhenshan, Wu Di, Yang Ding. Willingness to move, place dependence and community identity: An investigation of residential choice in the Zhongguancun area in Beijing. Progress in Geography, 2019, 38(3): 417-427. ]

[17] 吴翔华, 陈昕雨, 袁丰. 南京市住房困难人群职住关系 及影响因素分析 [J]. 地理科学进展, 2019, 38(12): 1890-1902. [Wu Xianghua, Chen Xinyu, Yuan Feng. Jobhousing relationship of people with housing difficulties and influencing factors in Nanjing City. Progress in Geography, 2019, 38(12): 1890-1902. ]

[18] 周春山. 改革开放以来大都市人口分布与迁居研究: 以 广州市为例 [D]. 广州: 中山大学, 1994. [Zhou Chunshan. A case study on the distribution and relocation of metropolitan population since reform and opening-up: A case study of Guangzhou. Guangzhou, China: Sun Yatsen University, 1994. ]

[19] 柴彦威, 陈零极. 中国城市单位居民的迁居: 生命历程
方法的解读 [J]. 国际城市规划, 2009, 24(5): 7-14. [Chai Yanwei, Chen Lingji. The residential mobility of urban Danwei residents: A life course approach. Urban Planning International, 2009, 24(5): 7-14. ]

[20] 古杰, 周素红, 用小培. 生命历程视角下的广州市居民 居住迁移的时空路径 [J]. 地理研究, 2013, 32(1): 157165. [Gu Jie, Zhou Suhong, Yan Xiaopei. The space-time paths of residential mobility in Guangzhou from a perspective of life course. Geographical Research, 2013, 32 (1): 157-165. ]

[21] 刘望保, 间小培. 转型期广州市居住迁移影响因素于户 籍之间的比较 [J]. 地理研究, 2007, 26(5): 1055-1066. [Liu Wangbao, Yan Xiaopei. Comparison of influencing factors for residential mobility between different household register types in transitional urban China: A case study of Guangzhou. Geographical Research, 2007, 26 (5): 1055-1066. ]

[22] 刘望保, 间小培, 曹小曙. 广州城市内部居住迁移空间 特征及其影响因素研究 [J]. 人文地理, 2007, 96(4): 2732. [Liu Wangbao, Yan Xiaopei, Cao Xiaoshu. The spatial characteristics and determinants of intra- urban residential mobility in Guangzhou. Human Geography, 2007, 96(4): 27-32. ]

[23] 王宇凡, 冯健. 基于生命历程视角的郊区居民迁居行为 重构: 以北京回龙观居住区为例 [J]. 人文地理, 2013, 28(3): 34-41. [Wang Yufan, Feng Jian. Restructuring of suburban residents' intra-urban migration behavior based on life course: A case study of Huilongguan Community in Beijing. Human Geography, 2013, 28(3): 34-41. ]

[24] Cui C, Geertman S, Hooimeijer P. The intra-urban distribution of skilled migrants: Case studies of Shanghai and Nanjing [J]. Habitat International, 2014, 44: 1-10.

[25] Cui C. Housing career disparities in urban China: A comparison between skilled migrants and locals in Nanjing [J]. Urban Studies, 2020, 57(3): 546-562.

[26] Kim J H, Pagliara F, Preston J. The intention to move and residential location choice behaviour[J]. Urban Studies, 2005, 42(9): 1621-1636.

[27] 张文忠, 刘旺. 西方城市居住区位决策与再选择模型的 评述 [J]. 地理科学进展, 2004, 23(1): 89-95. [Zhang Wenzhong, Liu Wang. The comment on research of western intra-urban residential relocation models. Progress in Geography, 2004, 23(1): 89-95. ]

[28] 张文忠. 城市居民住宅区位选择的因子分析 [J]. 地理 科学进展, 2001, 20(3): 267-274. [Zhang Wenzhong. An analysis of the factors that influence the urban residential location selection. Progress in Geography, 2001, 20(3): 267-274. ]

[29] 杜德斌, 崔裴, 刘小玲. 论住宅需求、居住选址与居住分 异 [J]. 经济地理, 1996, 16(1): 82-90. [Du Debin, Cui 
Pei, Liu Xiaoling. Housing demand, residential location and residential differentiation. Economic Geography, 1996, 16(1): 82-90. ]

[30] 张文忠, 刘旺, 李业锦. 北京城市内部居住空间分布与 居民居住区位偏好 [J]. 地理研究, 2003, 22(6): 751759. [Zhang Wenzhong, Liu Wang, Li Yejin. Housings' spatial distribution and residents' preference on housing location in Beijing. Geographical Research, 2003, 22(6): 751-759. ]

[31] 周素红, 刘玉兰. 转型期广州城市居民居住与就业地区 位选择的空间关系及其变迁 [J]. 地理学报, 2010, 65 (2): 191-201. [Zhou Suhong, Liu Yulan. The situation and transition of jobs-housing relocation in Guangzhou, China. Acta Geographica Sinica, 2010, 65(2): 191-201. ]

[32] Smith B, Olaru D. Lifecycle stages and residential location choice in the presence of latent preference heterogeneity $[\mathrm{J}]$. Environment and Planning A: Economy and Space, 2013, 45(10): 2495-2514.

[33] Lee B H Y, Waddell P. Residential mobility and location choice: A nested logit model with sampling of alternatives [J]. Transportation, 2010, 37(4): 587-601.

[34] Sermons M W, Koppelman F S. Representing the differences between female and male commute behavior in residential location choice models [J]. Journal of Transport Geography, 2001, 9(2): 101-110.

[35] Simpson W. Workplace location, residential location, and urban commuting [J]. Urban Studies, 1987, 24(2): 119-128.

[36] Chen J, Chen C, Timmermans H J P. Accessibility tradeoffs in household residential location decisions [J]. Transportation Research Record: Journal of the Transportation Research Board, 2008, 2077(1): 71-79.

[37] Wu W J, Zhang W Z, Dong G P. Determinant of residential location choice in a transitional housing market: Evidence based on micro survey from Beijing [J]. Habitat International, 2013, 39: 16-24.

[38] Prashker J, Shiftan Y, Hershkovitch-Sarusi P. Residential choice location, gender and the commute trip to work in Tel Aviv [J]. Journal of Transport Geography, 2008, 16 (5): 332-341.

[39] Tillema T, van Wee B, Ettema D. The influence of (tollrelated) travel costs in residential location decisions of households: A stated choice approach [J]. Transportation Research Part A: Policy and Practice, 2010, 44(10): 785796.

[40] Choocharukul K, Van H T, Fujii S. Psychological effects of travel behavior on preference of residential location choice [J]. Transportation Research Part A: Policy and Practice, 2008, 42(1): 116-124.

[41] Pinjari A R, Pendyala R M, Bhat C R, et al. Modeling the choice continuum: An integrated model of residential lo- cation, auto ownership, bicycle ownership, and commute tour mode choice decisions [J]. Transportation, 2011, 38 (6): 933-958.

[42] Schirmer P M, van Eggermond M A B, Axhausen K W. The role of location in residential location choice models: A review of literature [J]. Journal of Transport and Land Use, 2014, 7(2): 3-21.

[43] Fang Y P, Logan J R, Pal A. Emerging socio-spatial pattern of Chinese cities: The case of Beijing in 2006 [J]. Habitat International, 2015, 47: 103-112.

[44] Harold E, Leonard Z. Tenure choice, housing demand and residential location [J]. Journal of Real Estate Research, 1991, 6(3): 341-356.

[45] Yi C, Lee S. An empirical analysis of the characteristics of residential location choice in the rapidly changing Korean housing market [J]. Cities, 2014, 39: 156-163.

[46] Choudhury C F, Ayaz S B. Why live far? Insights from modeling residential location choice in Bangladesh [J]. Journal of Transport Geography, 2015, 48: 1-9.

[47] Źróbek S, Trojanek M, Źróbek-Sokolnik A, et al. The influence of environmental factors on property buyers' choice of residential location in Poland [J]. Journal of International Studies, 2015, 8(3): 164-174.

[48] Friedman J. A conditional logit model of the role of local public services in residential choice [J]. Urban Studies, 1981, 18(3): 347-358.

[49] Bailey A J. Population geography: Lifecourse matters [J]. Progress in Human Geography, 2009, 33(3): 407-418.

[50] Findlay A, McCollum D, Coulter R, et al. New mobilities across the life course: A framework for analysing demographically linked drivers of migration [J]. Population, Space and Place, 2015, 21(4): 390-402.

[51] Geist C, McManus P A. Geographical mobility over the life course: Motivations and implications [J]. Population, Space and Place, 2008, 14(4): 283-303.

[52] 谭一洺, 柴彦威, 杨永春. 基于 “推一拉”理论的城市少 数民族居民迁居动因探讨 [J]. 南京社会科学, 2017(3): 64-70, 80. [Tan Yiming, Chai Yanwei, Yang Yongchun. Motivation of migration with ethnic minority groups in the city based on pull-push theory: A case study in Lanzhou. Nanjing Journal of Social Sciences, 2017(3): 6470, 80. ]

[53] 赵莹, 柴彦威, 陈洁, 等. 时空行为数据的 GIS 分析方法 [J]. 地理与地理信息科学, 2009, 25(5): 1-5. [Zhao Ying, Chai Yanwei, Chen Jie, et al. GIS-based analyzing method for spatial- temporal behavior data. Geography and Geo-Information Science, 2009, 25(5): 1-5. ]

[54] 林荣平, 周素红, 间小培. 1978 年以来广州市居民职住 地选择行为时空特征与影响因素的代际差异 [J]. 地理 学 报, 2019, 74(4): 753- 769. [Lin Rongping, Zhou 
Suhong, Yan Xiaopei. Intergenerational differences of spatio-temporal characteristics and influencing factors of home-work location changes since 1978: Empirical analysis based on the micro-survey in Guangzhou, China. Acta Geographica Sinica, 2019, 74(4): 753-769. ]

[55] Huang Y Q, Yi D C, Clark W A V. Multiple home ownership in Chinese cities: An institutional and cultural perspective [J]. Cities, 2020, 97: 102518. doi: 10.1016/j.cities.2019.102518.

[56] Mok D. Do two-earner households base their choice of residential location on both incomes? [J]. Urban Studies, 2007, 44(4): 723-750.

[57] Cui C, Geertman S, Hooimeijer P. Residential mobility of skilled migrants in Nanjing, China [J]. Environment and Planning A: Economy and Space, 2015, 47(3): 625-642.

[58] 王春兰, 杨上广.上海人口郊区化与新城发展动态分析 [J]. 城市规划, 2015, 39(4): 65-70. [Wang Chunlan, Yang Shangguang. Dynamic analysis on population suburbanization and new town development of Shanghai City. City Planning Review, 2015, 39(4): 65-70. ]

[59] 王德, 朱查松, 谢栋灿. 上海市居民就业地迁移研究: 基 于手机信令数据的分析 [J]. 中国人口科学, 2016(1): 80-89, 127. [Wang De, Zhu Chasong, Xie Dongcan. Research on intra-city employment mobility in Shanghai: Based on cell phone data. Chinese Journal of Population Science, 2016(1): 80-89, 127. ]

\title{
Patterns and determinants of location choice in residential mobility: A case study of Shanghai
}

\author{
CUI Can ${ }^{1,2,3}$, MU Xueying ${ }^{2,3}$, CHANG Heying ${ }^{3}$, LI Jiayi ${ }^{3}$, WANG Fenglong ${ }^{1,2,4^{*}}$ \\ (1. Research Centre for China Administrative Division, East China Normal University, Shanghai 200241, China; \\ 2. The Centre for Modern Chinese City Studies, East China Normal University, Shanghai 200062, China; \\ 3. School of Urban and Regional Science, East China Normal University, Shanghai 200241, China; \\ 4. School of Geography, South China Normal University, Guangzhou 510631, China)
}

\begin{abstract}
Since the marketization of China's housing system, urban residents' housing adjustment through making residential moves has become relatively frequent. Residential mobility, as the micro-mechanism of urban space differentiation and restructuring, has been extensively studied in urban geography and housing studies. However, the existing literature mainly focuses on the motivation underlying residential mobility and its impacts on individuals/families and urban space. Comparatively, the location changes before and after residential moves have received scant attention in previous studies. This study adopted the perspective of life course and time geography to depict the residential trajectories of Shanghai residents and explore the influencing factors of location choice in residential mobility. The data used for the empirical analysis were drawn from the 2018 "Shanghai Resident Housing and Living Space Survey", which adopted the stratified and multi-stage probability proportion to size sampling. A retrospective survey was conducted, allowing us to obtain information on the respondents' sociodemographic information and their residential trajectories. The results reveal that the dominant type of location change is outward move across the ring roads. Nevertheless, the variations in location choice between cohorts, local population and migrants, and renters and owners of properties are evident. Compared with the older cohorts, younger cohorts generally make residential moves at earlier ages, and many of them move from the central areas to the suburbs. Different from the local population, migrants' residential mobility is more constrained in terms of the timing of making residential moves and their location choice. Furthermore, this study shows that age, location of workplace, and housing tenure all significantly affect location choice in making residential moves. Specifically, the older cohorts concentrate in the central areas before as well as after a residential move. Commuting distance plays a major role in affecting people's choice of residential location, and owning an automobile has insignificant influence. A transition into homeownership is often associated with a change to an advantageous location.
\end{abstract}

Keywords: location choice; residential mobility; housing tenure; time geography; Shanghai 